\title{
Doenças inflamatórias intestinais no Hospital Universitário da Universidade Federal de Sergipe: manifestações extraintestinais
}

\author{
Intestinal inflammatory diseases in the Hospital Universitario of Universidade \\ Federal de Sergipe: extraintestinal manifestations
}

\author{
JÚLIO AUGUSTO DO PRADO TORRES ${ }^{1}$, RAQUEL MATOS DE SANTANA ${ }^{1}$, \\ FELIPE AUGUSTO DO PRADO TORRES ${ }^{1}$, ALEX RODRIGUES MOURA ${ }^{2}$, JUVENAL DA ROCHA TORRES NETO 3 \\ ${ }^{1}$ Acadêmico do curso de Medicina da Universidade Federal de Sergipe (UFS), São Cristóvão (SE), Brasil. \\ ${ }^{2}$ Médico residente do Serviço de Coloproctologia da UFS, São Cristóvão (SE), Brasil. ${ }^{3}$ Professor Doutor e Chefe do \\ Serviço de Coloproctologia da UFS, São Cristóvão (SE), Brasil.
}

TORRES JAP, SANTANA RM, TORRES FAP, MOURA AR, TORRES NETO JR. Doenças inflamatórias intestinais no Hospital Universitário da Universidade Federal de Sergipe: manifestações extraintestinais. Rev bras Coloproct, 2011;31(2): 115-119.

RESUMO: A doença inflamatória intestinal idiopática (DII) representa um grupo de condições inflamatórias crônicas, resultantes de ativação persistente e inadequada do sistema imune mucoso. Além dos sintomas intestinais característicos, as DII podem se manifestar através de uma série de manifestações extraintestinais (MEI). Objetivos: Avaliar a incidência das MEI das doenças inflamatórias intestinais no Hospital Universitário da Universidade Federal de Sergipe; diagnosticar as MEI das DII; instituir o tratamento adequado dos pacientes portadores dessas manifestações. Métodos: Foi aplicado um protocolo para diagnóstico das MEI; quando necessário os pacientes foram encaminhados para as respectivas especialidades. Resultados: Foram catalogados 49 pacientes portadores de DII; destes, $41(83,6 \%)$ apresentaram MEI. As MEI reumatológicas foram as mais frequentes, acometendo 35 pacientes. $O$ restante das MEI foram assim distribuídas: um caso de MEI dermatológica; um caso de MEI urológica; um caso MEI pneumológica; quatro casos de MEI oftalmológicas; oito casos de MEI hepáticas. Conclusões: As MEI têm alta incidência (I=83,6\%) entre os pacientes portadores de DII; as MEI tiveram incidência semelhante entre os pacientes portadores de retocolite ulceratica idiopática e de Crohn; 0 início das MEI foi mais comum após o diagnóstico da DII; a classe de MEI mais prevalente foi a reumatológica $(\mathrm{P}=\mathbf{7 1 , 4 \% )}$.

Palavras-chave: doença inflamatória intestinal; proctocolite; doenças do cólon; patologia; complicações. Trabalho realizado no Serviço de Coloproctologia do Hospital Universitário da Universidade Federal de Sergipe (UFS), São Cristóvão (SE), Brasil.
Fonte de financiamento: não há Conflito de interesse: nada a declarar 
Rev bras Coloproct Abril/Junho, 2011
Doenças inflamatórias intestinais no Hospital Universitário da Universidade Federal de

Sergipe: manifestações extraintestinais

Júlio Augusto do Prado Torres e Cols.
Vol. 31

$\mathbf{N}^{\circ} 2$

\section{INTRODUÇÃO}

A doença inflamatória intestinal idiopática (DII), representada pela doença de Crohn e pela retocolite ulcerativa, é um grupo de condições inflamatórias crônicas, resultantes de ativação persistente e inadequada do sistema imune mucoso. Essa desregulação do sistema imune manifesta-se através de uma elevação local de diversas citocinas, como TNFalfa, interferon-gama, IL-12, IL-13 e IL-17 ${ }^{1}$. A etiologia dessas patologias ainda não foi definitivamente esclarecida ${ }^{2,3}$.

DII constitue-se em um problema de saúde pública em muitos países. Importantes trabalhos retrospectivos sobre a epidemiologia da DII realizados, sobretudo, a partir de 1980, demonstraram que está havendo uma tendência mundial para o aumento da sua incidência ${ }^{4-8}$. Sua incidência tem aumentado nos países desenvolvidos, girando em torno de 50 a 70 casos/1.000.000 por ano ${ }^{9}$, e há evidente tendência de crescimento da incidência nos países em desenvolvimento como ocorre na América do Sul ${ }^{10,11}$.

Além dos sintomas intestinais característicos (como diarreia, sangue e muco nas fezes, tenesmo retal), as DII podem se manifestar através de uma série de manifestações extraintestinais (MEI) que podem passar despercebidas. Entre essas, pode-se citar: articulares, como artralgia e espondilite anquilosante; dermatológicas, como eritema nodoso e psoríase; oftalmológicas, como uveíte e episclerite; urológicas, como litíase renal, insuficiência renal e nefroesclerose; hepatobiliares, como colelitíase, esteatose e colangite esclerosante; pulmonares, como derrame pleural, broquiectasia e asma; e manifestações vasculares, como trombose venosa e vasculites ${ }^{5}$.

Atualmente, temos ambulatório de referência em DII no Hospital Universitário da Universidade Federal de Sergipe (HU/UFS), com um número significativo de pacientes. É importante ressaltar a escassez de dados epidemiológicos acerca dessa patologia, em nosso país, que passa despercebida pelos grandes levantamentos de saúde pública nacional. Dada a falta de informações sobre a ocorrência das DII no Brasil, é essencial que se construa um perfil demográfico e clínico dos pacientes com DII e que se avalie a ocorrência das MEI nos mesmos.

\section{OBJETIVO}

Avaliar a incidência das MEI das DII (retocolite ulceratica idiopática -RCUI - e doença de Crohn - DC), diagnosticando-as e instituindo o tratamento adequado dos pacientes portadores dessas manifestações.

\section{MÉTODOS}

\section{Casuística}

Este trabalho consistiu em um estudo descritivo prospectivo, do tipo coorte longitudinal, onde foram incluídos os pacientes atendidos no ambulatório de referência de DII do Serviço de Coloproctologia do HU/ UFS, no período de agosto de 2009 a janeiro de 2010.

\section{Critérios de inclusão e exclusão}

Foram incluídos todos os pacientes portadores de DII, acompanhados no ambulatório de referência, no período de agosto de 2009 a janeiro de 2010.

Foram excluídos pacientes que abandonaram o acompanhamento ambulatorial ou que não tiveram dados completos para a pesquisa no prontuário.

\section{Métodos}

Foi aplicado um questionário para avaliar a ocorrência de MEI nos pacientes portadores de DII. Pacientes portadores de MEI foram encaminhados aos ambulatórios especializados para abordagem multidisciplinar.

\section{Considerações éticas}

O projeto foi submetido à apreciação do Comitê de Ética e Pesquisa da UFS. Todos os pacientes foram esclarecidos quanto aos objetivos da pesquisa e assinaram o Termo de Consentimento Livre e Esclarecido (Anexo2). Esta pesquisa não oferece riscos, nem gastos adicionais ao sujeito da pesquisa. $\mathrm{O}$ anonimato do entrevistado foi respeitado, bem como lhe foi assegurado o direito de não responder ao questionário.

\section{Análise estatística}

Os dados coletados foram lançados em um sistema de banco de dados informatizado e, posteriormente, os mesmos foram submetidos à análise estatística, utilizando-se o software Statistics Package of the So- 
cial Science (SPSS/PC+) versão 9.0 (1998). Foi feita uma análise estatística descritiva.

\section{RESULTADOS}

Foi catalogado um total de 49 pacientes portadores de DII no ambulatório de referência do HU/UFS, no período de agosto de 2009 a junho de 2010. Destes, $18(36,7 \%)$ eram portadores de DC e $31(63,2 \%)$ eram portadores de RCUI.

As MEI acometeram $41(83,6 \%)$ dos 49 pacientes. Desses 41 pacientes, $15(36,6 \%)$ eram portadores de DC e $26(63,4 \%)$ eram portadores de RCUI. Dos 8 pacientes que não apresentaram MEI, 5 (62,5\%) eram portadores de RCUI, e $3(37,5 \%)$ portadores de DC.

$\mathrm{O}$ início das MEI se deu antes do início da manifestação da DII em 10 (24,3\%) casos, concomitante ao aparecimento das manifestações da DII em 2 (4,8\%) casos e após o início das manifestações da DII em 29 $(70,7 \%)$ casos.

Houve 35 pacientes $(71,4 \%)$ com MEI reumatológica. Destes, 28 pacientes $(80 \%)$ com artralgia isolada; 1 paciente $(2,85 \%)$ com sacroileíte; 1 paciente $(2,85 \%)$ com sacroileíte e artralgia concomitantes; 4 pacientes $(11,4 \%)$ com artralgia e artrite concomitantes; e 1 paciente $(2,85 \%)$ com artralgia e espondilite anquilosante concomitantes. Subdividimos ainda as artralgias em axial/apendicular e pequenas/grandes articulações. Trinta e um pacientes $(91,1 \%)$ tiveram artralgias apendiculares e $3(8,9 \%)$ tiveram artralgias mistas (apendicular e axial); 1 (2,9\%) teve artralgia em pequenas articulações; 16 (47,0\%), artralgia de grandes articulações; 17 (50,0\%) tiveram um acometimento misto das articulações (grandes e pequenas).

Foi detectado apenas 1 caso $(2,0 \%)$ de MEI dermatológica: uma onicomicose secundária à imunossupressão do tratamento da DII.

Foram detectadas $3(6,1 \%)$ MEI vasculares: $2(66,6 \%)$ casos de trombose venosa profunda e 1 $(33,3 \%)$ caso de vasculite.

Foram detectadas $4(8,1 \%)$ MEI oftalmológicas: $2(50,0 \%)$ casos de conjuntivite e $2(50,0 \%)$ casos de glaucoma.

Foi detectado apenas $1(2,0 \%)$ MEI urológica: 1 caso de nefrolitíase.

Foram detectados $8(16,3 \%)$ casos de MEI hepáticas: $3(37,5 \%)$ casos de colelitíase; $3(37,5 \%)$ casos de esteatose hepática; $2(25,0 \%)$ casos de colangite esclerosante.

Foram detectados apenas $2(4,0 \%)$ casos de MEI pneumológica: 2 casos de asma.

\section{DISCUSSÃO}

As DII, representadas pela doença de Crohn e pela retocolite ulcerativa, resultam de uma ativação persistente e inadequada do sistema imune mucoso. $\mathrm{O}$ quadro clínico típico dessas entidades é expresso classicamente por uma diarreia invasiva, dor abdominal, náuseas e vômitos. No entanto, existem manifestações que não estão diretamente relacionadas ao sistema digestório, sendo assim chamadas de MEI, as quais são o foco da presente pesquisa.

Segundo Rogler et al. ${ }^{12}$, as MEI chegam a acometer mais da metade dos pacientes portadores de DII. De fato, nossa casuística apresentou uma incidência compatível com a literatura, tendo sido encontradas MEI em 83,6\% dos pacientes. Segundo Lakatos et $a .^{13}$, as MEI são mais comuns entre os portadores de doença de Crohn. Entretanto, os resultados obtidos vão de encontro à literatura, onde dos 41 portadores das MEI, 15 (36,6\%) tinham o diagnóstico de doença de Crohn e $26(63,4 \%)$ de retocolite. Porém, se esse dado de incidência absoluta for avaliado sob um prisma de relatividade, pode-se perceber que dos 18 pacientes com doença de Crohn, 15 (83,3\%) apresentaram MEI, e que dos 31 pacientes com Retocolite, $26(83,3 \%)$ apresentaram MEI. Sendo assim, foi obtido um resultado semelhante entre as duas formas de DII. Isso, somado ao fato de que tivemos um viés de amostragem, onde a maior parte dos pacientes eram portadores de retocolite, pode explicar o porquê de um dado conflitante com a literatura.

As MEI podem ser divididas em: reumatológicas, dermatológicas, vasculares, oftalmológicas, urológicas, hepáticas e pneumológicas. Segundo Lakatos et al. ${ }^{13}$ e Brynskov e Binder ${ }^{14}$, a artalgia é a MEI mais comum nas DII, tendo uma incidência que varia entre 10 e 35\%. Foi obtido um resultado que, apesar de concordante com a literatura, ficou superestimado, sendo encontrada uma incidência de $59 \%$ de artralgia isolada. As artralgias foram classificadas por Orchard et al. ${ }^{15}$ em tipo 1 (grandes articulações, pauciarticular e com evolução paralela à DII) e tipo 2 (peque- 
nas articulações, poliarticular e independente da DII). Ainda que a artralgia seja a principal representante em termos de número, vale destacar que também houve quatro casos de artrite, um de espondilite anquilosante e dois de sacroileíte. A casuística obtida foi mais compatível com o tipo 1 de Orchard.

Tavarela ${ }^{16}$ relatou uma incidência média de MEI cutâneas em torno de $10 \%$. O único caso de manifestação cutânea foi uma onicomicose secundária à imunossupressão do tratamento. Outras manifestações cutâneas, como eritema nodoso e pioderma gangrenoso, que são relativamente comuns segundo Tavarela $^{16}$, não foram encontradas em nenhum paciente. Essas manifestações são relacionadas com períodos de maior atividade da DII. Sendo assim, como os pacientes do serviço são acompanhados por um tempo longo e, portanto, têm um maior controle da DII, podem não ter tido a chance de evoluir com essas doenças.

As MEI oftalmológicas, raras segundo Mintz et al. ${ }^{17}$, não chegam a acometer $10 \%$ dos portadores de DII. Esse dado foi bem semelhante ao obtido na nossa casuística, com uma incidência de $8,1 \%$ da MEI oftalmológicas. Foram encontrados dois casos de conjuntivite e dois casos de glaucoma.

Derek $^{18}$ relatou que, entre as MEI urinárias/urológicas, a nefrolitíase é a manifestação mais comum, com uma incidência que pode chegar a $20 \%$ dos casos de doença de Crohn. No serviço, foi encontrado apenas um caso de nefrolitíase que, no entanto, ocorreu em um portador de retocolite. Nenhum caso de insuficiência renal foi relatado.

Raj e Lichenstein ${ }^{19}$ relatam que a manifestação hepatobiliar de relevância clínica mais frequente é a colangite esclerosante. Outra manifestação também importante é a colelitíase, que se correlaciona com lesão ileal. Encontramos $3(37,5 \%)$ casos de colelitíase, $3(37,5 \%)$ casos de esteatose e $2(25,0 \%)$ casos de colangite esclerosante. Os casos de colelitíase apenas ocorreram em portadores de doença de Crohn em 1 dos 3 casos, não havendo, portanto, correlação etiológica com doença ileal. Por outro lado, os 2 casos de colangite esclerosante ocorreram em porta- dores de retocolite ulcerativa, o que foi corroborado pela literatura.

Segundo $\operatorname{Mota}^{20}$, a incidência de eventos tromboembólicos nas DII é maior que na população geral, e a DII é reconhecida por proporcionar um estado de hipercoagulabildade, tanto na microvascularização do intestino inflamado, quanto em eventos sistêmicos, aumentando a morbidade e a mortalidade desses pacientes. A incidência de tromboembolismo nos portadores de DII varia de $2-10 \%$, o que é três a quatro vezes maior que na população geral. Entre as manifestações vasculares, o tromboembolismo foi de fato a patologia mais frequente, com uma taxa de incidência de $4,0 \%$. Além disso, também encontramos um caso de vasculite.

Mahadeva et al. ${ }^{21}$ apontam a bronquiectasia, a bronquiolite e a doença intersticial como as principais formas de acometimento pulmonar. Não foram enontradas nenhuma dessas manifestações entre os pacientes do nosso serviço. Foi encontrado apenas um caso de asma.

Após o levantamento desses pacientes com MEI das DII, temos, como objetivo, estabelecer, em todas as consultas de seguimentos dos pacientes, um questionário capaz de alertar para a ocorrência das mesmas e uma rotina de diagnóstico de tratamento mais adequado. Sendo a doença inflamatória intestinal uma doença de abordagem multidisciplinar, outras frentes de trabalho, como avaliação nutricional e psicológica dos mesmos.

\section{CONCLUSÃO}

As MEI têm alta incidência $(I=83,6 \%)$ entre os pacientes portadores de DII e as mais frequentes foram as reumatológicas, com uma prevalência de $71,4 \%$. As principais MEI encontradas na nossa série foram: artralgia, trombose venosa profunda, conjuntivite, glaucoma, colelitíase, esteatose hepática, colangite esclerosante e nefrolitíase. As MEI foram mais frequentes entre os pacientes portadores de RCUI. Seu início foi mais comum após o diagnóstico da DII. 
ABSTRACT: Idiopathic inflammatory bowel disease (IBD) represents a group of chronic inflammatory conditions, resulting from persistent and inappropriate activation of mucosal immune system. Besides the typical intestinal symptoms, the IBD can manifest itself through a series of extraintestinal manifestations (EIM). Objectives: To evaluate the incidence of EIM in the Hospital Universitario of Universidade Federal de Sergipe; to diagnose EIM of IBD; to institute the appropriate treatment of patients with these manifestations. Methods: We applied a protocol for diagnosis of EIM; when necessary, patients were referred to their specialities. Results: We categorized 49 patients with IBD, whose $41(83.6 \%)$ had EIM. The rheumatologics EIM were the most frequent, affecting 35 patients. The others EIM were distributed as follows: one case of dermatological EIM; one case of urologic EIM, one case of pulmonology EIM, four cases of ophthalmic EIM; eight cases of hepatic EIM. Conclusions: The EIM have a high incidence (I=83.6\%) among patients with IBD; the EIM had similar incidence among patients with ulcerative colitis and Crohn's disease. The beginning of EIM was more common after the diagnosis of IBD; the rheumatologic EIM was most prevalent $(\mathrm{P}=\mathbf{7 1 . 4 \%})$.

Keywords: inflammatory bowel disease; proctocolitis; colonic diseases; patolhogy; complications.

\section{REFERÊNCIAS}

1. Pinho M. A biologia molecular das doenças inflamatórias intestinais. Rev bras Coloproct 2008;28(1):119-23.

2. Jewel DP. Ulcerative colitis. In: Feldman M, Scharschimdt BF, Sleisenger MH, editors. Sleisenger \& Fordtran's gastrointestinal and liver disease: pathophysiology, diagnosis and management. $6^{\mathrm{a}}$ ed. Philadelphia: WB Saunders; 1998.

3. Kronbluth A, Sachar DK, Salomon P. Crohn's disease. In: Feldman M, Scharschimdt BF, Sleisenger MH, editors. Sleisenger \& Fordtran's gastrointestinal and liver disease: pathophysiology, diagnosis and management. $6^{\mathrm{a}}$ ed. Philadelphia: WB Saunders; 1998.

4. Farrokhyar F, Swarbrick ET, Irvine EJ. A critical review of epidemiological studies in inflammatory bowel disease. Scand J Gastroenterol 2001;36(1):2-15.

5. Ekbom A, Helmick C, Zack M, Adami HO. The epidemiology of inflammatory bowel disease: a large, population-based study in Sweden. Gastroenterology 1991;100(2):350-8.

6. Sedlack RE, Whisnant J, Elveback LR, Kurland LT. Incidence of Crohn's disease in Olmsted County, Minnesota, 19351975. Am J Epidemiol 1980;112(6):759-63.

7. Sonnenberg A. Geographic variation in the incidence of and mortality from inflammatory bowel disease. Dis Colon Rectum 1986;29(12):854-61.

8. Stonnington CM, Phillips SF, Melton LJ 3rd, Zinsmeister AR. Chronic ulcerative colitis: incidence and prevalence in a community. Gut 1987;28(4):402-9.

9. Logan RF. Inflammatory bowel disease incidence: up, down or unchanged? Gut 1998;42(3):309-11.

10. Biondo-Simões MLP, Mandelli KK, Pereira MSC, Faturi JL. Opções terapêuticas para as doenças inflamatórias intestinais: revisão. Rev bras Coloproct 2003;23(3):172-82.

11. Steinwurz F. Epidemiologia, aspectos clínicos e evolutivos da doença de Crohn. Arq Gastroenterol 1998;35:237-9.

12. Rogler G, Schölmerich J. Extraintestinal manifestations of inflammatory bowel disease. Med Klin (Munich)
2004;99(3):123-30.

13. Lakatos L, Pandur T, David G, Balogh Z, Kuronya P, Tollas A, et al. Associaton of extraintestinal manifestations of inflammatory bowel disease in province of Western Hungary with disease phenotype: Results of 25-year follow-up study. World J Gastroenterology 2003;9(10): 2300-7.

14. Brynskov J, Binder V. Arthritis and the gut. Eur J Gastroenterol Hepatol 1999;11(9):997-9.

15. Orchard TR, Wordsworth BP, Jewell DP. Peripheral arthropathies in inflammatory bowel disease: their articular distribution and natural history. Gut 1998;42(3):387-91.

16. Tavarela Veloso F. Review article: skin complications associated with inflammatory bowel diseases. Aliment Pharmacol Ther 2004;20(4):50-4.

17. Mintz R, Feller ER, Bahr RL, Shah SA. Ocular manifestations of inflammatory bowel disease. Inflamm Bowel Dis 2004;10(2):135-9.

18. Derek PJ. Ulcerative colitis. In: Feldman M, Friedman LS, Sleisenger MS, editors. Gastrointestinal and liver disease: pathophysiology, diagnosis, management. $7^{\mathrm{a}}$ ed. Philadelphia: Saunders; 2002.

19. Raj V, Lichenstein DR. Hepatobiliary manifestations of inflammatory bowel disease. Gastroenterol Clin North Am 1999;28(2):491-513.

20. Mota ES. Manifestações extra-intestinais em doença de Crohn e retocolite ulcertiva: prevalência e correlação com o diagnóstico, extensão, atividade, tempo de evolução da doença. Dissertação. São Paulo: Faculdade de Medicina, Universidade de São Paulo; 2007.

21. Mahadeva R, Walsh G, Flower CD, Shneerson JM. Clinical and radiological characteristics of lung disease in inflammatory bowel disease. Eur Respir J 2000;15(1):41-8.

Endereço para correspondência:

Juvenal da Rocha Torres Neto

Rua Ananias Azevedo, 100, apto. 902 - Praia 13 de Julho

CEP 49020-080 - Aracaju (SE), Brasil.

E-mail: jtorres@infonet.com.br 